\title{
Modelling the Effect of Treatment and Infected Immigrants on the Spread of Hepatitis C Virus Disease with Acute and Chronic Stages
}

\author{
Neterindwa Ainea ${ }^{1}$, Estomih S. Massawe ${ }^{1, *}$, Oluwole Daniel Makinde ${ }^{2}$ \\ ${ }^{1}$ Mathematics Department, University of Dar es Salaam, P. O. Box 35062, Dar es Salaam, Tanzania \\ ${ }^{2}$ Institute for Advance Research in Mathematical Modelling and Computations, Cape-Peninsula University of technology, P. O. Box 1906, \\ Bellville 7535, South Africa
}

\begin{abstract}
This paper examines the effect of Treatment and Infected Immigrants on the spread of Hepatitis C Virus (HCV) disease with Acute and Chronic stages. A nonlinear mathematical model for the problem is proposed and analysed qualitatively using the stability theory of the differential equations. The results show that the disease free equilibrium is locally stable at threshold parameter less than unity and unstable at threshold parameter greater than unity. Globally, the disease free equilibrium is not stable due existence of forward bifurcation at threshold parameter equal to unity. However the disease becomes more endemic due to the presence of infected immigrants in the community. It is also shown that in the presence of treatment, the rate of infected immigrants (acute and chronic) decreases and consequently the treated infected individuals decreases continuously. Numerical simulation of the model is implemented to investigate the sensitivity of certain key parameters on the treatment and infected immigrants on the spread of the disease with acute and chronic stages.
\end{abstract}

Keywords Treatment Of HCV, Infectious Disease, Infections By Immigrants, Spread Of Infectious Disease

\section{Introduction}

Hepatitis (plural hepatitides) is an inflammation of the liver characterized by the presence of inflammatory cells in the tissue of the organ[1]. The inflammation of liver causes soreness and swelling. Hepatitis is most commonly caused by one of the 5 hepatitis viruses; hepatitis $A$, hepatitis B, hepatitis $C$, hepatitis $D$ and hepatitis E. Hepatitis $C$ is usually spread through contact with blood products[2]. Blood products have been the main agents through which HCV is transmitted, but ever since 1992, when it became possible to detect the virus in blood, transmissions through transfusions, and organ transmissions have been minimal.

Most common avenues through which HCV is spread are unprotected sex, sharing of contaminated needles among drug addicts and those with other STDs[3]. Some people also get this virus from tattoo and piercing salons. It is also possible to contract $\mathrm{HCV}$ at birth, as it can be transmitted from mother to baby.

A HCV infection can be categorized into two stages, firstly an acute infection (following initial infection) and secondly a chronic infection. Acute means 'new 'or illness of

* Corresponding author:

emassawe@uccmail.co.tz (Estomih S. Massawe)

Published online at http://journal.sapub.org/ajcam

Copyright (C) 2012 Scientific \& Academic Publishing. All Rights Reserved a short period. A chronic hepatitis $\mathrm{C}$ infection is when the infection lasts for longer than six months.

The goal of treatment is to reduce the amount of the HCV in the blood to levels that can't be detected after 24 weeks of therapy. HCV is treated with drugs that slow or stop the virus from damaging the liver. The treatment of HCV has advanced in recent years which have greatly improved the outlook for people with HCV. The usual treatment of HCV is a combination of two different medicines called pegylated interferon and ribavirin[2].

[4] made an analysis on the immigration status, race and language barriers on chronic hepatitis virus infection management and treatment outcomes. The researchers found that $\mathrm{HCV}$ endemic regions of the word are diagnosed with $\mathrm{HCV}$ infection after immigration and subsequently referred to viral hepatitis chronics for disease management. They suggested that optimal care to all patients, indentifying and understanding barriers to care related to immigrant status, race and language in $\mathrm{HCV}$ management must be provided.

[5] found that chronic $\mathrm{HCV}$ is a progressive condition that accounts for at least one quarter of all cases of chronic liver disease[6] predicted that the prevalence of chronic $\mathrm{HCV}$ (CH-C) remains high and the complications of infection are common[7] discovered that chronic HCV complications are increasing, especially among people older than 60 years[8] conducted a study on disease progression of Acute HCV[9] analyzed the screening of $\mathrm{HCV}$ in a Health maintenance 
Organization[10], investigated the effects of a HCV educational intervention or a motivational intervention on alcohol use and sexual risk behaviours among injection drug users

[11] investigated the dynamic behaviour of an SEI (Susceptible- Exposed- Infective) model with acute and chronic stages. However the integration of treatment and infective immigrants to the population was not incorporated. In this paper, it is intended to study and analyze a model which incorporates the impact of treatment and infective immigrants on the spread of HCV transmission dynamics. Thus we study and analyse a deterministic model of the effect of treatment and infected immigrants on the spread of $\mathrm{HCV}$ disease with acute and chronic stages.

\section{Model Formulation}

A non linear mathematical model is proposed and analyzed to study the effect of treatment and infected immigrants on the spread of $\mathrm{HCV}$ disease with acute and chronic stages.

In modelling the dynamics, the population is divided into five groups: Susceptible individuals 'S'; Exposed individuals ' $\mathrm{E}$ ', who are not yet infectious; Acute infected class ' $I$ ' who are individuals initially infected; Chronic infected class ' $V$ ', who are infectious individuals; Treated group ' $T$ ' but not yet cured.

The interaction between the classes will be assumed as follows: Exposed $(E)$ acute infected $(I)$ and chronic infected $(V)$ immigrants enter into the population with the rates $\delta_{1}, \delta_{2}, \delta_{3}$, respectively. Susceptible individuals

are shifted to exposed class $(E)$ by the rate $\delta_{1}$. Also susceptible individuals $(S)$ may become acute infectious $(I)$ and chronic infectious $(V)$ shifted to Treatment class $(T)$ at the rates $\pi_{1}, \pi_{2}$ respectively. The number of new infectious individuals produced is therefore $\frac{\left(\beta_{1} I+\beta_{2} V+\beta_{3} T\right) S}{N}$ where $\beta_{1}, \beta_{2}, \beta_{3}$ are the effective contact rate of individuals with acute hepatitis $\mathrm{C}$ chronic hepatitis $\mathrm{C}$, and the individual still undergoing Treatment but not yet cured respectively.

In formulating the model, the following assumptions are taken into consideration:

i. Both treatment $(T)$ and chronic infectious individuals

$(V)$ recover with the constant rates $\rho>0$ and $\alpha>0$ respectively,

ii. The recovered individuals go back to susceptible $(S)$

group, iii. The rate of transmission is directly proportional to the susceptible population and also to the ratio between the infected population and the total population. Total population at time $t$ is given by

$$
N(t)=S(t)+E(t)+I(t)+T(t)+V(t),
$$

iv. Following the interactions of susceptible, exposed class, acute individuals, chronic infective and treated group in everyday activities, susceptible acquire HCV infection with the force of infection $v$,

v. The population is homogeneously mixed and each susceptible individual has equal chance of acquiring $\mathrm{HCV}$ infection when he/she comes into contact with infectious individual,

vi. The population under study comprise of persons with at least fifteen (15) years old. In this case, it is assumed that there is no vertical transmission and all recruitments are immigrants.

vii. All parameters in population are positive and all compartments suffer natural mortality at a rate $\mu$.

Taking into account the above considerations, we then have the following schematic flow diagram (Figure1):

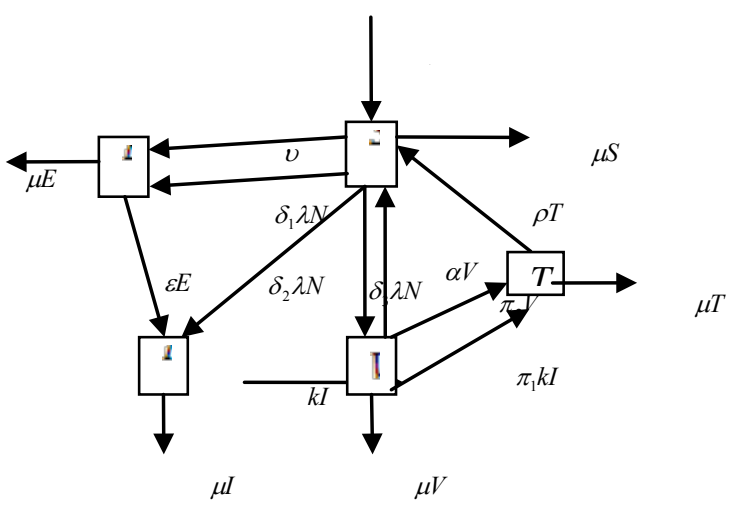

Figure 1. Model flowchart.

From the above flow chart, and with

$$
v=\frac{\left(\beta_{1} I+\beta_{2} V+\beta_{3} T\right)}{N} S,
$$

the model will be governed by the following system of equations:

$$
\begin{gathered}
\frac{d S}{d t}=\left(1-\delta_{1}-\delta_{2}-\delta_{3}\right) \lambda N-\frac{\left(\beta_{1} I+\beta_{2} V+\beta_{3} T\right) S}{N} \\
+\alpha V+\rho T-\mu S
\end{gathered}
$$

$$
\frac{d E}{d t}=\delta_{1} \lambda N+\frac{\left(\beta_{1} I+\beta_{2} V+\beta_{3} T\right) S}{N}-(\varepsilon+\mu) E
$$

$$
\begin{aligned}
& \frac{d I}{d t}=\delta_{2} \lambda N+\varepsilon E-(k+\mu) I \\
& \frac{d T}{d t}=\pi_{1} k I+\pi_{2} V-(\rho+\mu) T
\end{aligned}
$$




$$
\frac{d V}{d t}=\delta_{3} \lambda N+\left(1-\pi_{1}\right) k I-\left(\pi_{2}+\alpha+\mu\right) V
$$

with nonnegative initial conditions and $N(0)>0$. where

$\beta_{i}(\mathrm{i}=1,2,3)$ are the effective contact rates of individuals with acute hepatitis $\mathrm{C}$, chronic hepatitis $\mathrm{C}$, and the individual still undergoing treatment but not yet cured,

$\delta_{i}(\mathrm{i}=1,2,3)$ are the rates at which exposed $(E)$, acute infected $(I)$ and chronic infected $(V)$ immigrants enter into the population respectively,

$\lambda$ is the recruitment rate,

$\mu$ is the death rate,

$k$ is the rate of progression from acute infected class to both treatment and chronic infected class,

$\varepsilon$ is the rate of progression from the exposed to acute infected class,

$\pi_{1}, \pi_{2}$ are the rates of progression from acute infected and chronic infected groups to treatment respectively,

$\rho, \alpha$ are the rates of progression for treatment from acute infected and chronic infected group respectively

Since the model is homogeneous of degree one, the variables can be normalized by setting $s=S / N, e=E / N$, $i=I / N, h=T / N, v=V / N$. This leads to the normalized system

$$
\begin{gathered}
\frac{d s}{d t}=\left(1-\delta_{1}-\delta_{2}-\delta_{3}-s\right) \lambda \\
-\left(\beta_{1} i+\beta_{2} v+\beta_{3} h\right)_{s}+\alpha v+\rho h \\
\frac{d e}{d t}=\left(\delta_{1}-e\right) \lambda+\left(\beta_{1} i+\beta_{2} v+\beta_{3} h\right) s-\varepsilon e \\
\frac{d i}{d t}=\left(\delta_{2}-i\right) \lambda+\varepsilon e-k i \\
\frac{d h}{d t}=\pi_{1} k i+\pi_{2} v-(\rho+\lambda) h \\
\frac{d v}{d t}=\left(\delta_{3}-v\right) \lambda+\left(1-\pi_{1}\right) k i-\pi_{2} v-\alpha v
\end{gathered}
$$

\section{Model Analysis}

The nonlinear system in Equation (1) will be qualitatively analyzed so as to find the conditions for existence and stability a disease free equilibrium points[12]. Analysis of the model allows us to determine the effect of treatment with infected immigrants on the spread of $\mathrm{HCV}$ disease with acute and chronic stages. Also on finding the reproductive number $R_{0}$, one can determine if the disease become endemic in a population or not.

\subsection{Disease Free Equilibrium (DFE)}

The disease free equilibrium of the normalised model system of equations ( 3 ) is obtained by setting

$$
\frac{d s}{d t}=\frac{d e}{d t}=\frac{d i}{d t}=\frac{d h}{d t}=\frac{d v}{d t}=0 .
$$

At disease free equilibrium we have $s=1, \quad e=i=h=v=0$ Thus the system (3) becomes

$$
\lambda-\lambda s=0 \text {. }
$$

Therefore the disease free equilibrium (DFE) denoted by $\epsilon_{0}$ of the normalised model system (3) is given by

$$
\epsilon_{0}=(s, 0,0,0,0)=(1,0,0,0,0) .
$$

\subsection{Local Stability of Disease Free Equilibrium}

The disease free equilibrium of the normalised model system (3) was given by

$$
€_{0}=(s, 0,0,0,0)=(1,0,0,0,0) .
$$

The local stability of $€_{0}$ is established by using the next generation operator method on the normalised model system (3). The basic reproduction number $R_{0}$ is defined as the effective number of secondary infections caused by typical infected individual during his/her entire period of infectiousness[13]. This definition is given for the models that represent spread of infection in a population. It is obtained by taking the largest (dominant) Eigen value, (spectral radius) of

$$
\left[\frac{\partial F_{i}\left(€_{0}\right)}{\partial X_{j}}\right] \cdot\left[\frac{\partial V_{i}\left(€_{0}\right)}{\partial X_{j}}\right]^{-1}
$$

where

$F_{i}$ is the rate of appearance of new infection in compartment $i$,

$V_{i}$ is the transfer of individuals out of the compartment $i$ by all other means,

$€_{0}$ is the disease free equilibrium.

Therefore

$$
F_{i}=\left(\begin{array}{c}
F_{1} \\
F_{2} \\
F_{3} \\
F_{4}
\end{array}\right)=\left(\begin{array}{c}
\left(\beta_{1} i+\beta_{2} v+\beta_{3} h\right) s \\
0 \\
0 \\
0
\end{array}\right)
$$

By linearization approach, the associated matrix at disease free equilibrium is obtained as

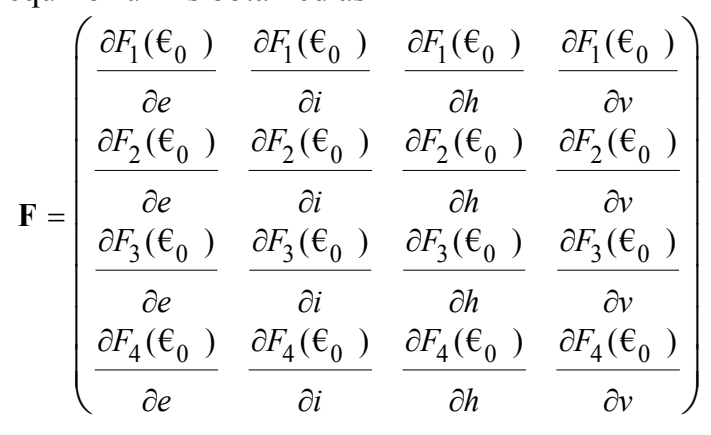


This gives

$$
\mathbf{F}=\left(\begin{array}{cccc}
0 & \beta_{1} & \beta_{3} & \beta_{2} \\
0 & 0 & 0 & 0 \\
0 & 0 & 0 & 0 \\
0 & 0 & 0 & 0
\end{array}\right)
$$

The transfer of individuals from the compartment $i$ is given by

$$
V_{i}=\left(\begin{array}{c}
V_{1} \\
V_{2} \\
V_{3} \\
V_{4}
\end{array}\right)=\left(\begin{array}{c}
(\varepsilon+\lambda) e-\lambda \delta_{1} \\
(k+\lambda) i-\delta_{2} \lambda-\varepsilon e \\
(\rho+\lambda) h-\pi_{1} k i-\pi_{2} v \\
\left(\pi_{2}+\alpha+\lambda\right) v-\delta_{3} \lambda-k i\left(1-\pi_{1}\right)
\end{array}\right)
$$

Again, by linearization approach, the associated matrix at disease free equilibrium is given by

$$
\mathbf{V}=\left(\begin{array}{llll}
\frac{\partial V_{1}\left(\epsilon_{0}\right)}{\partial e} & \frac{\partial V_{1}\left(\epsilon_{0}\right)}{\partial i} & \frac{\partial V_{1}\left(\epsilon_{0}\right)}{\partial h} & \frac{\partial V_{1}\left(\epsilon_{0}\right)}{\partial v} \\
\frac{\partial V_{2}\left(\epsilon_{0}\right)}{\partial e} & \frac{\partial V_{2}\left(\epsilon_{0}\right)}{\partial i} & \frac{\partial V_{2}\left(\epsilon_{0}\right)}{\partial h} & \frac{\partial V_{2}\left(\epsilon_{0}\right)}{\partial v} \\
\frac{\partial V_{3}\left(\epsilon_{0}\right)}{\partial e} & \frac{\partial V_{3}\left(\epsilon_{0}\right)}{\partial i} & \frac{\partial V_{3}\left(\epsilon_{0}\right)}{\partial h} & \frac{\partial V_{3}\left(\epsilon_{0}\right)}{\partial v} \\
\frac{\partial V_{4}\left(\epsilon_{0}\right)}{\partial e} & \frac{\partial V_{4}\left(\epsilon_{0}\right)}{\partial i} & \frac{\partial V_{4}\left(\epsilon_{0}\right)}{\partial h} & \frac{\partial V_{4}\left(\epsilon_{0}\right)}{\partial v}
\end{array}\right)
$$

This gives

$$
\mathbf{V}=\left(\begin{array}{cccc}
\varepsilon+\lambda & 0 & 0 & 0 \\
-\varepsilon & k+\lambda & 0 & 0 \\
0 & -\pi_{1} k & \lambda+\rho & -\pi_{2} \\
0 & -\left(1-\pi_{1}\right) k & 0 & \alpha+\pi_{2}+\lambda
\end{array}\right)
$$

With

$$
\mathbf{v}^{-1}=\left(\begin{array}{ccc}
\frac{1}{\theta+\delta_{1}+\lambda} & 0 & 0 \\
\frac{\theta}{\left(\theta+\delta_{1}+\lambda\right)\left(\gamma_{1}+\delta_{2}+\lambda\right)} & \frac{1}{\left(\gamma_{1}+\delta_{2}+\lambda\right)} & 0 \\
\frac{\theta \gamma_{1}}{\left(\theta+\delta_{1}+\lambda\right)\left(\gamma_{1}+\delta_{2}+\lambda\right)(\sigma+\lambda)} & \frac{\gamma_{1}}{\left(\gamma_{1}+\delta_{2}+\lambda\right)(\sigma+\lambda)} & \frac{1}{\sigma+\lambda}
\end{array}\right)
$$

Therefore

$$
\mathbf{F V}^{-1}=\left(\begin{array}{cccc}
M & N & R & Q \\
0 & 0 & 0 & 0 \\
0 & 0 & 0 & 0 \\
0 & 0 & 0 & 0
\end{array}\right)
$$

Where

$$
\begin{gathered}
M=\frac{\beta_{1} \varepsilon}{(\varepsilon+\lambda)(k+\lambda)}+\frac{\beta_{3} k \varepsilon\left(\pi_{1} \alpha+\pi_{1} \lambda+\pi_{2}\right)}{(\varepsilon+\lambda)(k+\lambda)(\lambda+\rho)\left(\alpha+\pi_{2}+\lambda\right)} \\
-\frac{\beta_{2}\left(-1+\pi_{1}\right) k \varepsilon}{(\varepsilon+\lambda)(k+\lambda)\left(\alpha+\pi_{2}+\lambda\right)} \\
N=\frac{\beta_{1}}{(k+\lambda)}+\frac{\beta_{3} k\left(\pi_{1} \alpha+\pi_{1} \lambda+\pi_{2}\right)}{(k+\lambda)(\lambda+\rho)\left(\alpha+\pi_{2}+\lambda\right)} \\
-\frac{\beta_{2}\left(-1+\pi_{1}\right) k}{(k+\lambda)\left(\alpha+\pi_{2}+\lambda\right)} \\
R=\frac{\beta_{3}}{(\lambda+\rho)}
\end{gathered}
$$

and

$$
Q=\frac{\beta_{3} \pi_{2}}{(\lambda+\rho)\left(\alpha+\pi_{2}+\lambda\right)}+\frac{\beta_{2}}{\alpha+\pi_{2}+\lambda}
$$

The Eigen values of $\mathbf{F V}^{-1}$ are $(0,0,0, Z)$ where

$$
\begin{gathered}
Z=\frac{\beta_{1} \varepsilon}{(\varepsilon+\lambda)(k+\lambda)}+\frac{\beta_{3} k \varepsilon\left(\pi_{1} \alpha+\pi_{1} \lambda+\pi_{2}\right)}{(\varepsilon+\lambda)(k+\lambda)(\lambda+\rho)\left(\alpha+\pi_{2}+\lambda\right)} \\
-\frac{\beta_{2}\left(-1+\pi_{1}\right) k \varepsilon}{(\varepsilon+\lambda)(k+\lambda)\left(\alpha+\pi_{2}+\lambda\right)}
\end{gathered}
$$

It follows that the basic reproduction number for the normalised model system (3) with infective immigrant and treatment denoted by $R_{0}$ is given by

$$
\begin{gathered}
R_{0}=\frac{\beta_{1} \varepsilon}{(\varepsilon+\lambda)(k+\lambda)} \\
+\frac{\beta_{3} k \varepsilon\left(\pi_{1} \alpha+\pi_{1} \lambda+\pi_{2}\right)}{(\varepsilon+\lambda)(k+\lambda)(\lambda+\rho)\left(\alpha+\pi_{2}+\lambda\right)} \\
-\frac{\beta_{2}\left(-1+\pi_{1}\right) k \varepsilon}{(\varepsilon+\lambda)(k+\lambda)\left(\alpha+\pi_{2}+\lambda\right)}
\end{gathered}
$$

The disease free equilibrium of the treatment model system (3) with infective immigrants is locally asymptotically stable if $R_{0}<1$ and unstable if $R_{0}>1$. In order to assess the contribution of $i, v$ and $h$ in terms of $\beta_{1}, \beta_{2}$ and $\beta_{3}$ respectively from equation (12) above, we let

$$
\begin{gathered}
R_{0 i}=\frac{\beta_{1} \varepsilon}{(\varepsilon+\lambda)(k+\lambda)}, \\
R_{0} v=-\frac{\beta_{2}\left(-1+\pi_{1}\right) k \varepsilon}{(\varepsilon+\lambda)(k+\lambda)\left(\alpha+\pi_{2}+\lambda\right)}, \\
R_{0 h}=\frac{\beta_{3} k \varepsilon\left(\pi_{1} \alpha+\pi_{1} \lambda+\pi_{2}\right)}{(\varepsilon+\lambda)(k+\lambda)(\lambda+\rho)\left(\alpha+\pi_{2}+\lambda\right)}
\end{gathered}
$$

Then

$$
R_{0}=R_{0 i}+R_{0 v}+R_{0 h}
$$

\section{Lemma 1}

The disease free equilibrium of the treatment model system (3) with infective immigrants is locally asymptotically stable if $R_{0}<1$ and unstable If $R_{0}>1$.

Remark:From the equations (14) above, it is clear that $R_{0 i}>R_{0 h}>R_{0 v}$ which implies that acute infective ' $i$ ' have a significant contribution on the transmission of the $\mathrm{HCV}$ infection followed by individuals who are still undergoing treatment but not yet cured ' $h$ ' which keep the disease endemic (i.e. $R_{0}>1$ ) in the population through " $\beta_{1}, \delta_{1} "$ and " $\beta_{3}, \delta_{3}$ " respectively compared to chronic infective ' $v$ ' under " $\beta_{2}, \delta_{2}$ ".

In the absence of infection, the population size approaches the steady state for a normalized model system (3). The results by[11] on $R_{0} \quad R_{0}$ are recovered only when $\beta_{3}=0$ and $\pi_{1}=\pi_{2}=0$ from equation (12) above. 


\subsection{Endemic Equilibrium and Local Stability}

To obtain an endemic equilibrium $E^{*}$, we set each equation in the model (3) equal to zero. Solving the system while expressing each equilibrium point in terms of $i^{*}$ at steady state, we get $s^{*}(t), e^{*}(t), i^{*}(t), h^{*}(t)$, and $v^{*}(t)$ as an endemic equilibrium point. Thus

$$
E^{*}=\left(\left(s^{*}(t)\right),\left(e^{*}(t)\right),\left(i^{*}(t)\right),\left(h^{*}(t)\right),\left(v^{*}(t)\right)\right)
$$

is an endemic equilibrium where

$$
\begin{gathered}
s^{*}=\frac{\left(r_{3}+r_{4} i^{*}\right)}{\lambda+R_{0} r_{1} i^{*}+r_{2}} \\
e^{*}=\frac{\delta_{1} \lambda\left(\lambda+R_{0} r_{1} i^{*}+r_{2}\right)+\left(R_{0} r_{1} i^{*}+r_{2}\right)\left(r_{3}+r_{4}{ }^{*}\right)}{\left(\lambda+R_{0} r_{1} i^{*}+r_{2}\right)(\lambda+\varepsilon)} \\
h^{*}=\frac{\left(\lambda \pi_{1}+\alpha \pi_{1}+\pi_{2}\right) k i^{*}+\pi_{2} \delta_{3} \lambda}{(\rho+\lambda)\left(\lambda+\pi_{2}+\alpha\right)} \\
v^{*}=\frac{\delta_{2} \lambda+\left(1-\pi_{1}\right) k i^{*}}{\lambda+\pi_{2}+\alpha}
\end{gathered}
$$

and

$$
\begin{gathered}
r_{1}=\frac{(\varepsilon+\lambda)(k+\lambda)}{\varepsilon}, r_{2}=\frac{\beta_{2} \delta_{3} \lambda(\rho+\lambda)+\beta_{3} \pi_{2} \delta_{3} \lambda}{(\rho+\lambda)\left(\lambda+\pi_{2}+\alpha\right)} \\
r_{3}=\frac{\lambda(\rho+\lambda)\left(\lambda+\pi_{2}+\alpha\right)\left(1-\delta_{1}-\delta_{2}-\delta_{3}\right)+(\rho+\lambda) \alpha \delta_{3} \lambda+\rho \pi_{2} \delta_{3} \lambda}{(\rho+\lambda)\left(\lambda+\pi_{2}+\alpha\right)} \\
r_{4}=\frac{(\rho+\lambda) \alpha\left(1-\pi_{1}\right) k+\rho\left(\lambda \pi_{1}+\alpha \pi_{1}+\pi_{2}\right) k}{(\rho+\lambda)\left(\lambda+\pi_{2}+\alpha\right)}
\end{gathered}
$$

We note that $h^{*}, \mathrm{v}^{*}, \mathrm{~s}^{*}, e^{*}$ are always positive and this will happen if and only if $R_{0}>0$.

\subsection{Global Stability of DFE}

Theorem 1: If $R_{0}>1$, the endemic equilibrium $E^{*}$ of the model (3) is globally asymptotically stable.

Proof: To establish the global stability of the endemic equilibrium $E^{*}$, we construct the following Lyapunov function:

$$
\begin{array}{r}
V\left(s^{*}, e^{*}, i^{*}, h^{*}, v^{*}\right)=\left(s-s^{*}-s^{*} \log \frac{s^{*}}{s}\right) \\
+\left(e-e^{*}-e^{*} \log \frac{e^{*}}{e}\right)+\left(i-i^{*}-i^{*} \log \frac{i^{*}}{i}\right) \\
+\left(h-h^{*}-h^{*} \log \frac{h^{*}}{h}\right)+\left(v-v^{*}-v^{*} \log \frac{v^{*}}{v}\right)
\end{array}
$$

By directly calculating the derivation of $V$ along the solution of (3) we get

$$
\frac{d V}{d t}=\left(\frac{s-s^{*}}{s}\right) \frac{d s}{d t}+\left(\frac{e-e^{*}}{e}\right) \frac{d e}{d t}
$$

$$
+\left(\frac{i-i^{*}}{i}\right) \frac{d i}{d t}+\left(\frac{h-h^{*}}{h}\right) \frac{d h}{d t}+\left(\frac{v-v^{*}}{v}\right) \frac{d v}{d t}
$$

which gives

$$
\frac{d V}{d t}=a-b
$$

Where

$$
\begin{gathered}
a=\left(\beta_{1}{ }^{*}+\beta_{2} v^{*}+\beta_{3} h^{*}\right) \frac{\left(s-s^{*}\right)^{2}}{s} \\
+\frac{s^{*}}{s}\left(\alpha v+\rho h+\beta_{1} i^{*} s+\beta_{2} v^{*} s+\beta_{3} h^{*} s\right) \\
+\left(\delta_{1}+\delta_{2}+\delta_{3}\right) \lambda \\
+\frac{e^{*}}{e}\left(\beta_{1} i^{*} s+\beta_{2} v^{*} s+\beta_{3} h^{*} s+\beta_{1} i s^{*}+\beta_{2} v s^{*}+\beta_{3} h s^{*}\right) \\
+\frac{h^{*}}{h}\left(\pi_{1} k i^{*}+\pi_{2} v^{*}\right)+\frac{v^{*}}{v}\left(k i^{*}+\pi_{1} k i\right) \\
+\alpha v+\rho h+\pi_{2} v+k i+\lambda+\lambda\left(\delta_{1}+\delta_{2}+\delta_{3}\right) \\
+\varepsilon e+\beta_{2} v s+\beta_{3} h s+\beta_{1} i s
\end{gathered}
$$

and

$$
\begin{gathered}
b=\left(\lambda+\beta_{1} i+\beta_{2} v+\beta_{3} h\right) \frac{\left(s-s^{*}\right)^{2}}{s} \\
+(\lambda+\varepsilon) \frac{\left(e-e^{*}\right)^{2}}{e}+(\lambda+k) \frac{\left(i-i^{*}\right)^{2}}{i} \\
+(\rho+\lambda) \frac{\left(h-h^{*}\right)^{2}}{h}+\left(\lambda+\pi_{2}+\alpha\right) \frac{\left(v-v^{*}\right)^{2}}{v} \\
+\frac{s^{*}}{s}\left(\lambda+\alpha v+\rho h+\beta_{2} v s+\beta_{3} h s+\beta_{1} i s\right) \\
+\frac{e^{*}}{e}\left(\delta_{1} \lambda+\beta_{1} i s+\beta_{2} v s+\beta_{3} h s+\beta_{1} i^{*} s^{*}+\beta_{2} v^{*} s^{*}+\beta_{3} h^{*} s^{*}+\varepsilon e\right) \\
+\frac{i^{*}}{i}\left(\delta_{2} \lambda+\varepsilon e+k i+\beta_{1} s i\right) \\
+\frac{h^{*}}{h}\left(\pi_{1} k i+\pi_{2} v+\rho h+\beta_{3} s h\right) \\
+\frac{v^{*}}{v}\left(k i+\pi_{1} k i^{*}+\delta_{3} \lambda+\alpha v++\pi_{2} v+\beta_{2} s v\right) \\
+\left(\delta_{1}+\delta_{2}+\delta_{3}\right) \lambda
\end{gathered}
$$

Thus from (14), if $a<b$ then $\frac{d V}{d t}$ will be negative definite, implying that $\frac{d V}{d t}<0$. Also $\frac{d V}{d t}=0$ if and only if $s=s^{*}, e=e^{*}, i=i^{*}, h=h^{*}, v=v^{*}$.

Therefore, the largest compact invariant set in 
$\left\{\left(s^{*}, e^{*}, i^{*}, h^{*}, v^{*}\right) \in \Gamma: \frac{d V}{d t}=0\right\}$ is the singleton $\left\{\mathrm{E}^{*}\right\}$, where $E^{*}$ is endemic equilibrium of the normalised system (3). By LaSalle's invariant principle, it then implies that $\mathrm{E}^{*}$ is globally asymptotically stable in $\Gamma$ if $a<b$.

\subsection{Determination of Forward or Backward Bifurcation}

The existence and stability of endemic equilibrium is determined through the investigation of the possibility of existence of the backward or forward bifurcation due to existence of endemic equilibrium using the the centre manifold theory [14]. As a disease invades the population, it reduces the number of susceptible individuals in the population, which tends to reduce its reproductive rates.

Figure (2) shows forward bifurcation for the chosen numerical data:

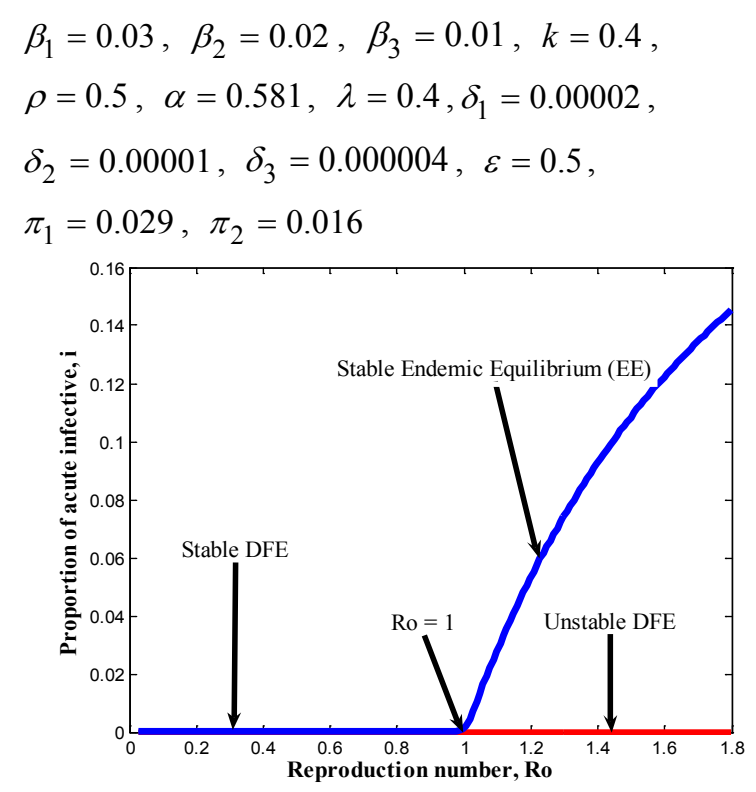

Figure 2. The forward or transcritical bifurcation.

A Forward or Transcritical bifurcation at the stationary solutions occurs at $R_{0}=1$. If $\mathrm{R}_{0}<1$, no biologically meaningful endemic stationary solution exists, and the disease free stationary solution is a global attractor. But if $R_{0}>1$, the endemic solution exists and it is a global attractor, while the disease free solution is a saddle point. This is referred to as a forward bifurcation because in the neighbourhood of the bifurcation point, the endemic disease prevalence is an increasing function of $R_{0}$.

\subsection{Model with Infected Immigrants and Treatment When Treated HCV Infectives Do Not Transmit the Infection}

$$
\left(\pi_{1} \neq 0, \pi_{2} \neq 0, \beta_{3} \neq 0\right)
$$

In this case, we consider the situation where the treated $\mathrm{HCV}$ infectives take the preventive measures and change their behaviour so as not to transmit the infection in the community $\left(\beta_{3}=0\right)$. Thus the infection is transmitted by acute and chronic infectives individuals only. The basic reproduction number in this case is given by

$$
R_{01}=\frac{\beta_{1} \varepsilon}{(\varepsilon+\lambda)(k+\lambda)}+\frac{\beta_{2}\left(1-\pi_{1}\right) k \varepsilon}{(\varepsilon+\lambda)(k+\lambda)\left(\alpha+\pi_{2}+\lambda\right)}
$$

and we note that $\mathrm{R}_{01}<R_{0}$. We also note that $\mathrm{R}_{0} \rightarrow \mathrm{R}_{01}$ as $\beta_{3} \rightarrow 0$. Therefore we can conclude that the endemicity of the infection is reduced when the treated HCV infectives show a positive attitude towards preventive measures and accelerate the transmission of the disease.

\subsection{Model in the Absence of Treatment}

$$
\left(\pi_{1}=0, \pi_{2}=0, \beta_{3}=0\right)
$$

Here we consider the situation where there is no treatment of the acute infectives $\left(\pi_{1}=0\right)$ and chronic infectives $\left(\pi_{2}=0\right)$. In this case the normalised model system (3) is reduced to the group of proportion of susceptibles, exposed individuals, acute infectives, and chronic infective individuals in the community. As there is no treatment, meaning that $\pi_{1}=0$ and $\pi_{2}=0$ it implies that $h \rightarrow 0$. We therefore obtain the basic reproduction number as

$$
R_{02}=\frac{\beta_{1} \varepsilon}{(\varepsilon+\lambda)(k+\lambda)}+\frac{\beta_{2} k \varepsilon}{(\varepsilon+\lambda)(k+\lambda)(\alpha+\lambda)}
$$

We note that $R_{02}<\mathrm{R}_{0}$ and $R_{0} \rightarrow R_{02}$ as $\pi_{1} \rightarrow 0, \pi_{2} \rightarrow 0, \beta_{3} \rightarrow 0$. Therefore it can be concluded that in the absence of treatment, the endemicity of the infection increases.

Considering the two epidemiological cases discussed above, it may be concluded that if both infected immigrants and treated HCV infectives take the preventive measures and do not spread the infection, then the disease tends to the endemic state if the rate of both infected immigrants and treated HCV infectives is small. If the annual infected immigrants and treatment rates are very high (say $100 \%$ of the initial population), the disease may tend to disease free equilibrium point. However if the infected immigrants (acute and chronic) and treated $\mathrm{HCV}$ infectives contribute to the transmission of the disease, then even if treatment rates are very high, the disease settles in the population as the system approaches asymptotically stable endemic equilibrium point. Analysis also shows that the endemicity of the disease is reduced by treatment of $\mathrm{HCV}$ infectives in the community.

\section{Numerical Simulations}

In order to verify the theoretical predictions of the model, the numerical simulations of the model (3) are carried out using the following set of estimated parameter values:

$\beta_{1}=3.5, \quad \beta_{2}=2, \quad \beta_{3}=0.2, \quad k=0.8, \quad \rho=0.4$, 
$\alpha=0.581, \quad \lambda=0.8, \quad \varepsilon=0.5, \quad \pi_{1}=0.6, \quad \pi_{2}=0.3$, $\delta_{1}=0.00002, \quad \delta_{2}=0.00001, \delta_{3}=0.000004$.

Figures 4.1-4.4 below show the proportion of exposed population and $\mathrm{HCV}$ infective populations (acute, treated and chronic infectives) plotted against the proportion of susceptible population. The figures show the dynamic behaviour of the endemic equilibrium of the normalized model (3) using the estimated parameter values above for different starting values in four cases as shown below:
$s(0)=0.6909$,
$e(0)=0.2$,
$i(0)=0.1$,
$h(0)=0.009$,
$v(0)=0.00001$
$s(0)=0.9$,
$e(0)=0.5$,
$i(0)=0.1$,
$h(0)=0.06$,
$v(0)=0.03$
$s(0)=0.4$,
$e(0)=0.2$,
$i(0)=0.09$,
$h(0)=0.03$,
$v(0)=0.01$
$s(0)=0.2$,
$e(0)=0.1$,
$i(0)=0.06$,
$h(0)=0.005$,
$v(0)=0.0005$

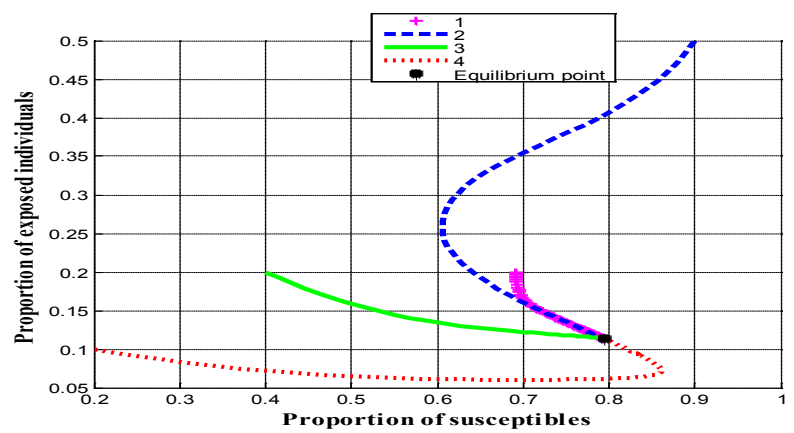

Figure 4.1. Variation of proportion of exposed individuals against proportion of susceptible population.

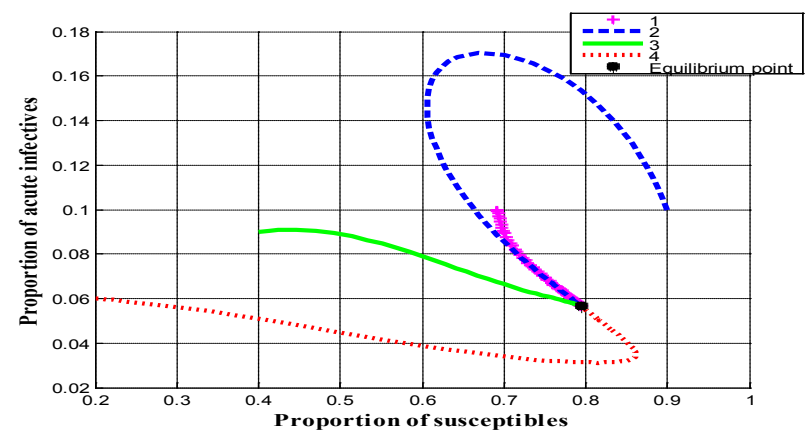

Figure 4.2. Variation of proportion of acute infective population against susceptible population.

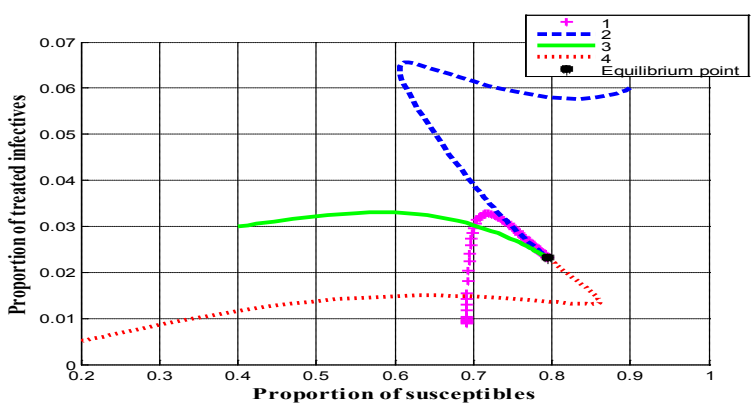

Figure 4.3. Variation of proportion of treated infective population against susceptible population.

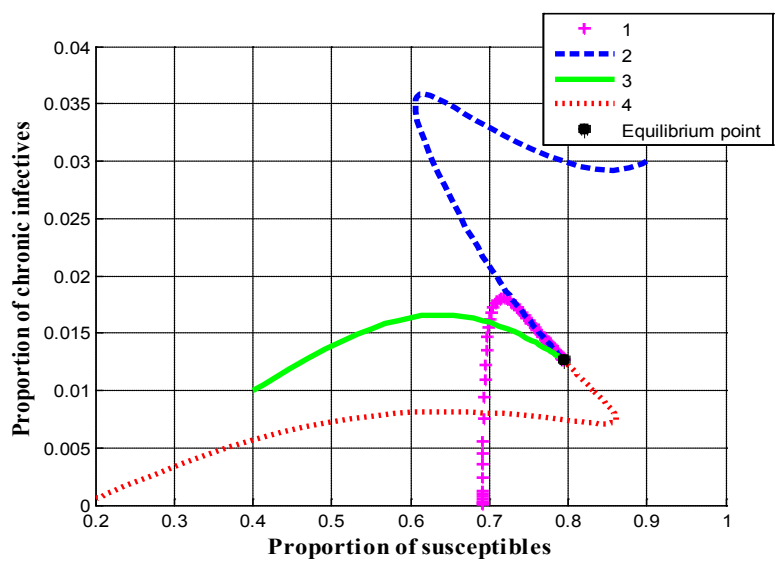

Figure 4.4. Variation of proportion of chronic infective population against susceptible population.

In figures 4.1-4.4, the endemic equilibrium $E^{*}$ was obtained as $s^{*}=0.7941, e^{*}=0.1134, i^{*}=0.05671$, $h=0.02321, v^{*}=0.01268$. It is can be observed from these figures that for any initial starting point, the solution curves tend to the endemic equilibrium point $E^{*}$. Hence, we infer that the system (3) is globally stable about the endemic equilibrium point $E^{*}$ for the set of parameters chosen.

In figure 4.5 below, the distribution of the proportion of population with time is shown in different classes without infective immigrant and treatment

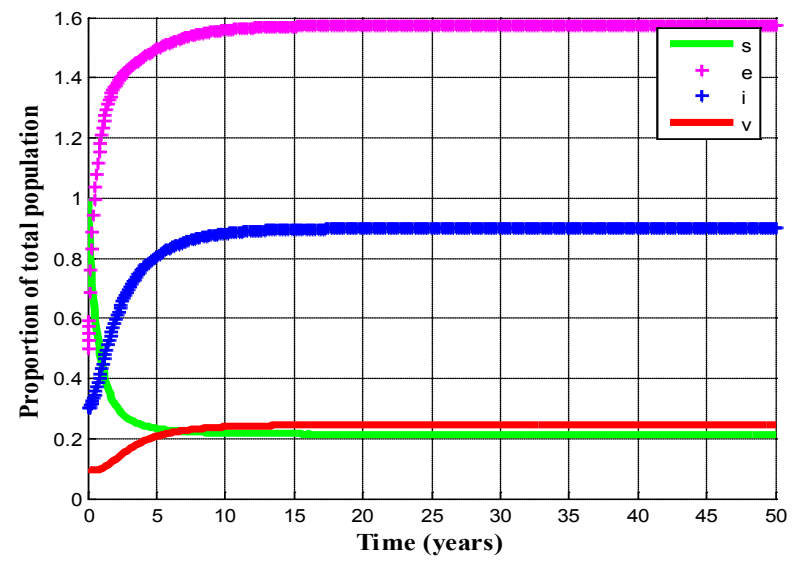

Figure 4.5. Variation of proportion of the population without infected immigrants and treatment.

It is observed that in the absence of HCV infected immigrants and treatment class in the community, the susceptible population decreases continuously which results in an increase in infective population (acute, chronic and treated infectives) and then it decreases as some of the infected population recovery naturally due to strong immune systems. Other infected individuals will die out by disease induced deaths due to absence of HCV treatment.

Figure. 4.6 below shows the distribution of proportion of population with time in all classes with the rates of infective immigrants $\delta_{i}(i=1,2,3)$ and the rate of treatment $\pi_{i}(i=1,2)$. 


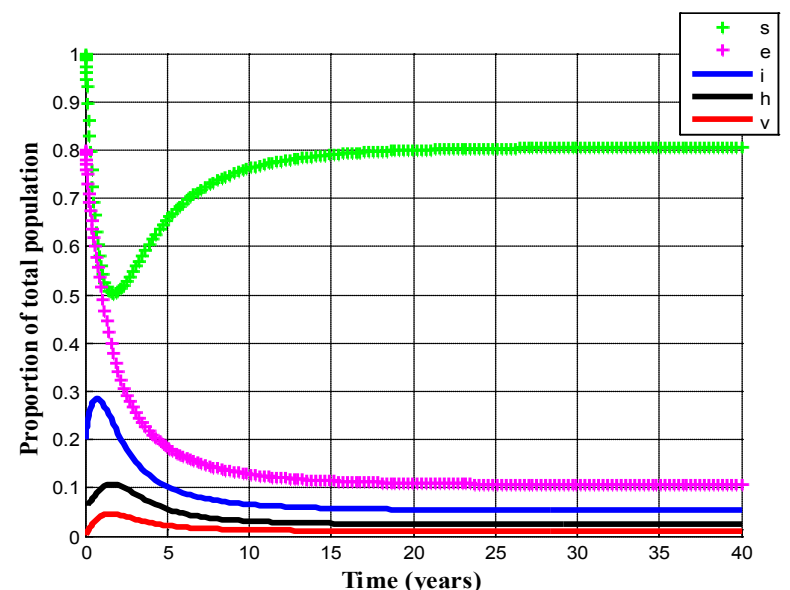

Figure 4.6. Variation of proportion of the population with infected immigrants and treatment.

It is observed that, initially the proportion of susceptible population decreases with time due to immigration of infected immigrants and then increases with time due to recovery rates of individuals $\rho, \alpha$ and eventually it reaches equilibrium position. Also the susceptible class decreases with time since individuals moved to the acute infectives at the rate $\varepsilon$. The acute infected population decreases with time since infected individuals moved to both treatment and chronic infected class. As the rate of treatment increases, the infective individuals decreases with time leading to the increase of susceptible individuals and reaches the equilibrium position.

Figure 4.7 below shows the variation of the proportion of susceptible individuals with different rates of infected immigrants $\delta_{i}(i=1,2,3)$

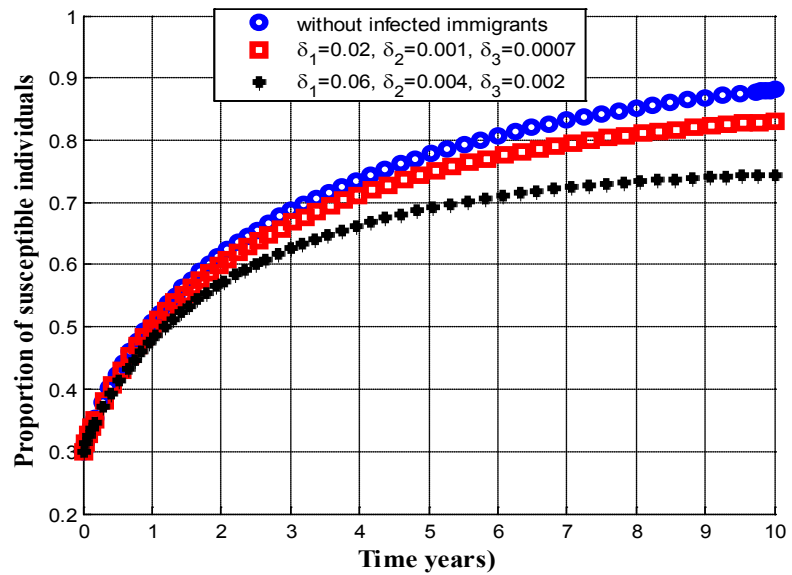

Figure 4.7. Variation of proportion of susceptible population for different values of $\delta_{i}(i=1,2,3)$

It is observed that as the infected immigrants increase, susceptible individuals decreases with time resulting in the increase of the number of infective individuals in the community.

Figs 4.8-4.11 below show the variation of proportion of exposed individuals, acute, chronic and treated infected populations respectively.

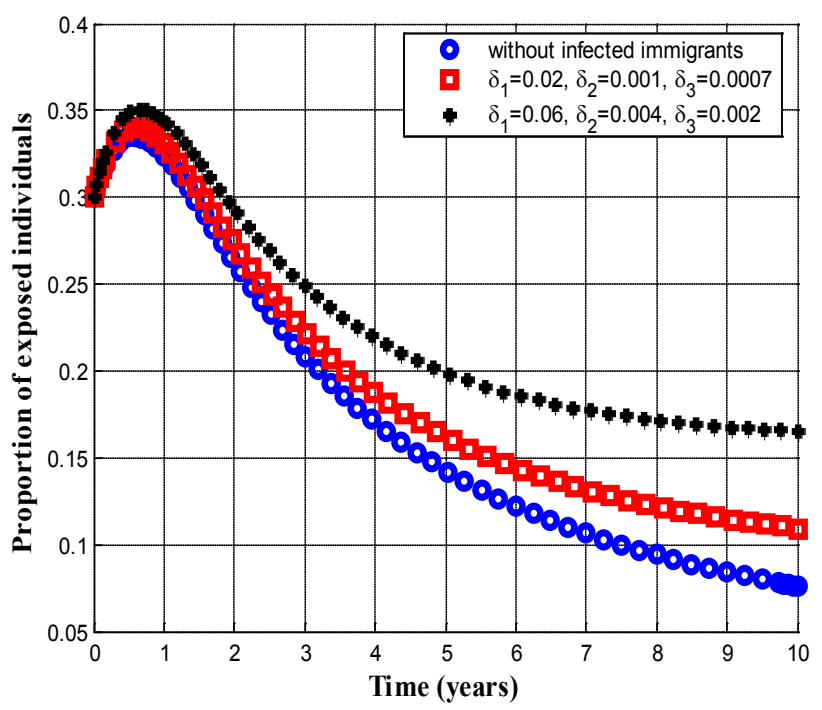

Figure 4.8. Variation of proportion of exposed individuals for different values of $\delta_{i}(i=1,2,3)$.

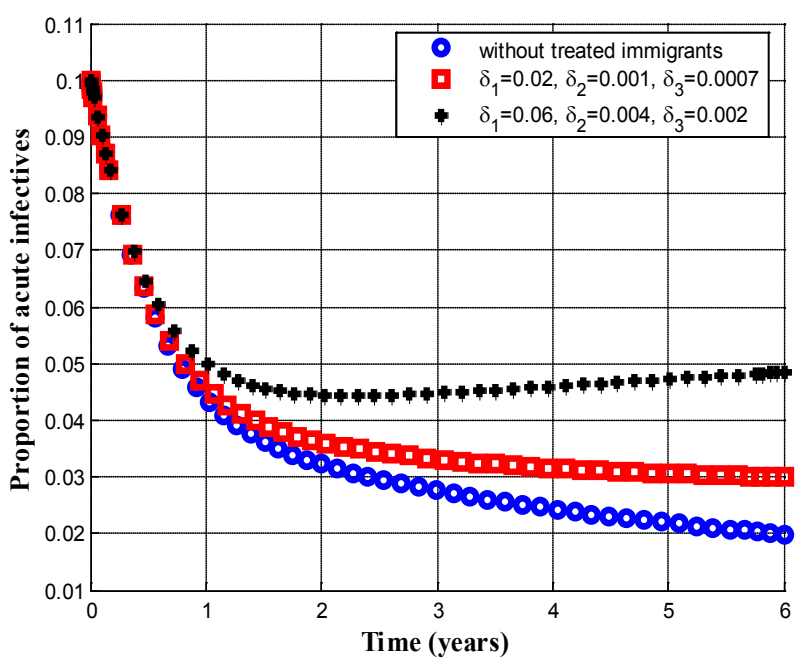

Figure 4.9. Variation of proportion of acute infective population for different values of $\delta_{i}(i=1,2,3)$.

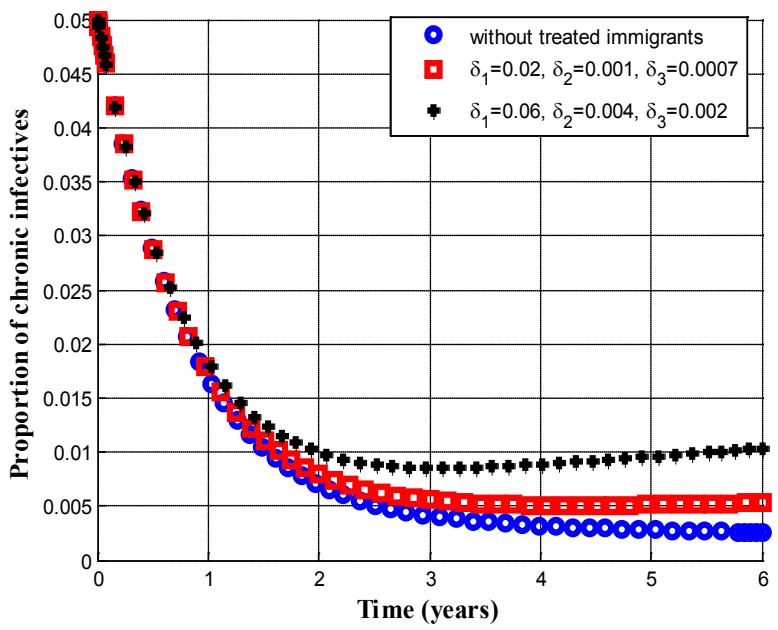

Figure 4.10. Variation of proportion of chronic infectives for different values of $\delta_{i}(i=1,2,3)$ 


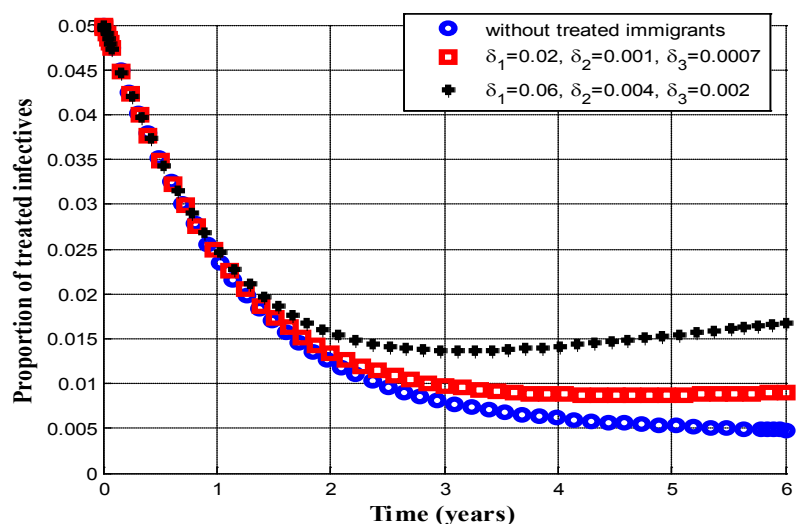

Figure 4.11. Variation of proportion of treated infected population for different values of $\delta_{i}(i=1,2,3)$.

It is observed from figure 4.8 that as the infected immigrants increase, the exposed individuals increase with time. Figure 4.9 shows that the exposed individuals shift to acute class at the rate $\varepsilon$ which results in the increase of the number of acute infective individuals. Then, the acute infected individuals shifts to both treatment and chronic infected class at the rate $k$, leading to the increase of the number of chronic infective individuals as seen in figure 4.10 and treated infective individuals as seen in figure 4.11.

Figures 4.12 and 4.13 show the variation of proportion of acute and chronic infectives for different values of $\pi_{i}(i=1,2)$

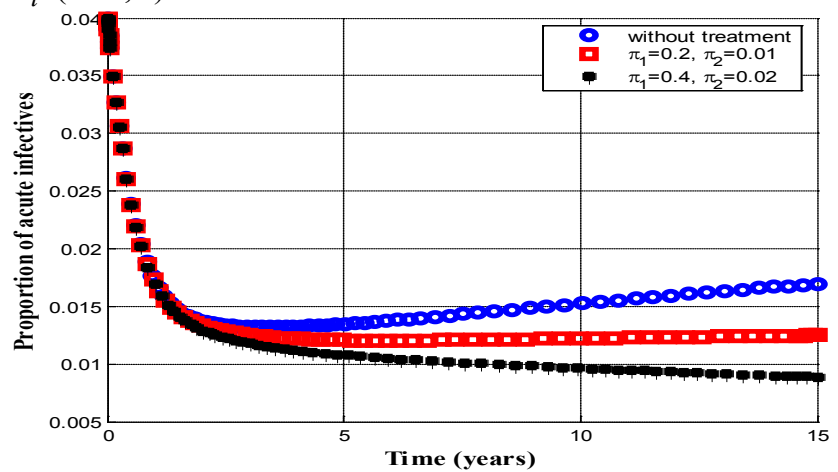

Figure 4.12. Variation of proportion of acute infective population for different values of $\pi_{i}(i=1,2)$.

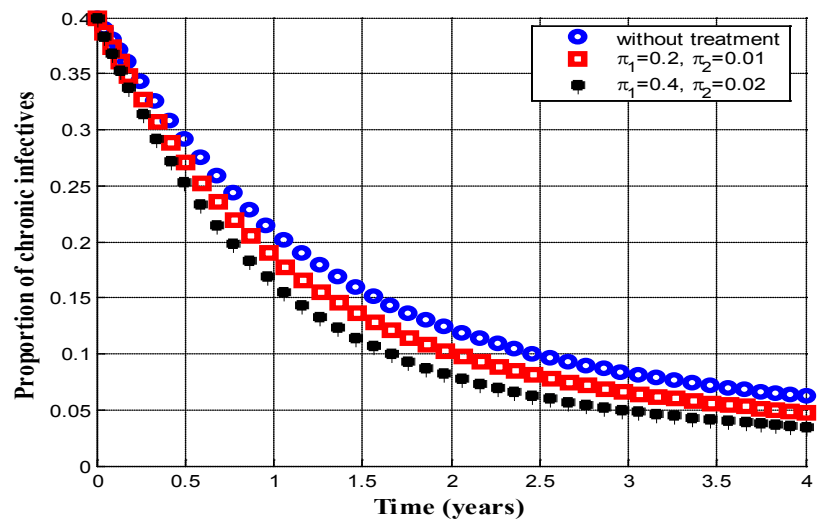

Figure 4.13. Variation of proportion of chronic infective population for different values of $\pi_{i}(i=1,2)$.
It is observed that the acute infective individuals decrease with time due to the increase of the rate of progression to treatment from acute infected individuals $\pi_{1}$ as seen in figure 4.12. Also as the rate of progression to treatment from chronic infectives $\pi_{1}$ increase, the chronic infected individuals decrease as seen in figure 4.13.

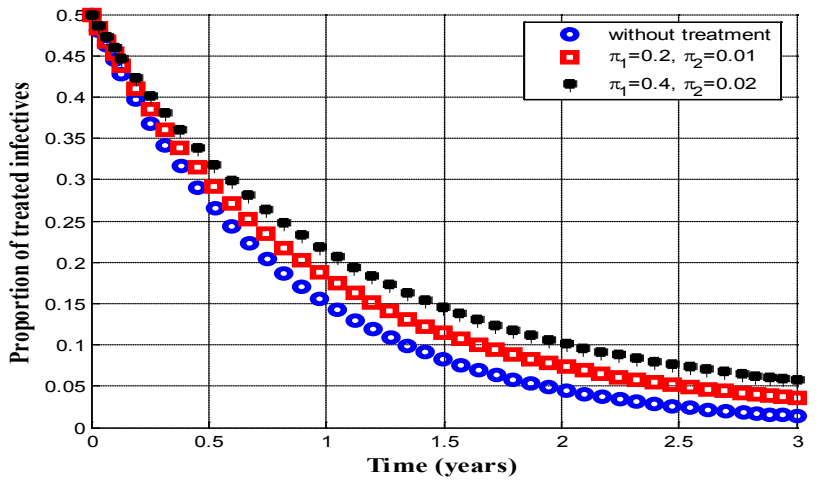

Figure 4.14. Variation of proportion of treated infectives for different values of $\pi_{i}(i=1,2)$.

In figure 4.14 , it is seen that the treated individuals increases with time due to the increase of the rate of progression to treatment from acute and chronic infectives $\pi_{1}$ and $\pi_{2}$ respectively.

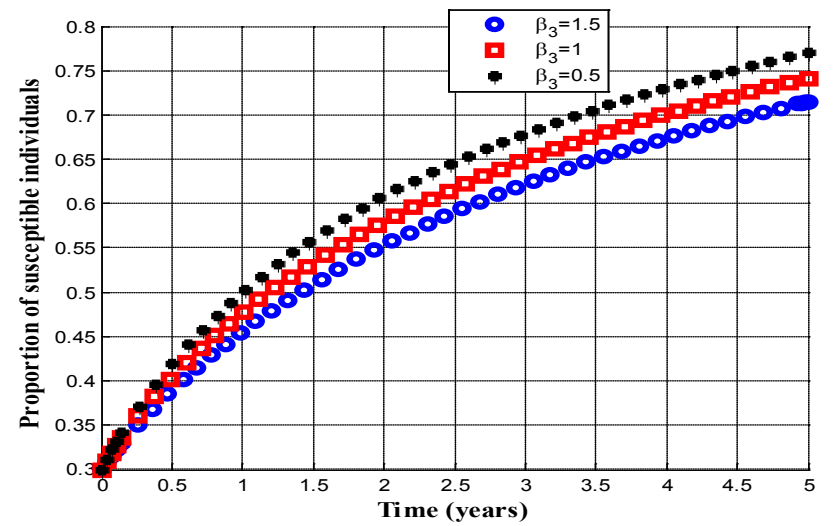

Figure 4.15. Variation of proportion of susceptible population for different values of $\beta_{i}(i=1,2,3)$.

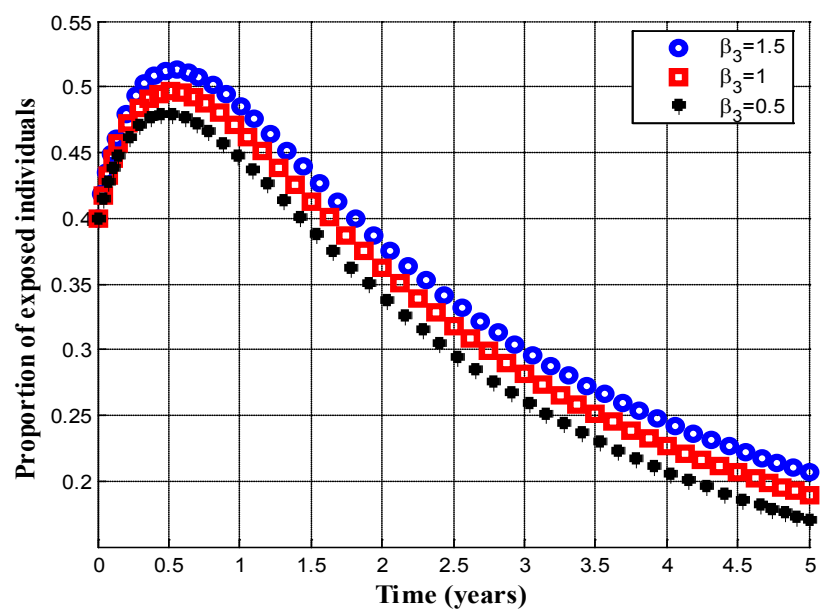

Figure 4.16. Variation of proportion of exposed individuals for different values of $\beta_{i}(i=1,2,3)$. 
It is observed from figures. 4.15 and 4.16 that when the effective contact rate of individuals still undergoing treatment $\beta_{3}$ become very small, the susceptible population increase with time.(see figure 4.15) and if the susceptible population increase, the number of exposed individuals decrease (see figure 4.16).

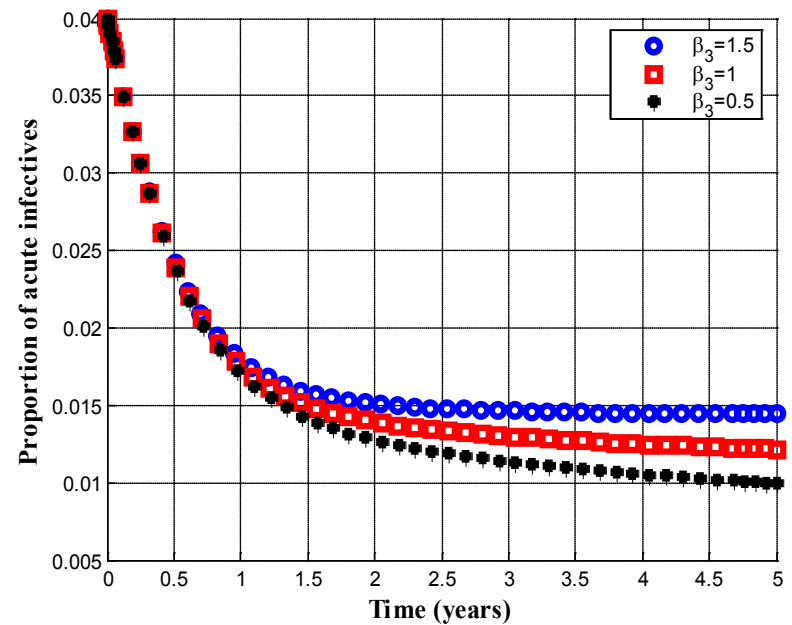

Figure 4.17. Variation of proportion of acute infective population for different values of $\beta_{i}(i=1,2,3)$.

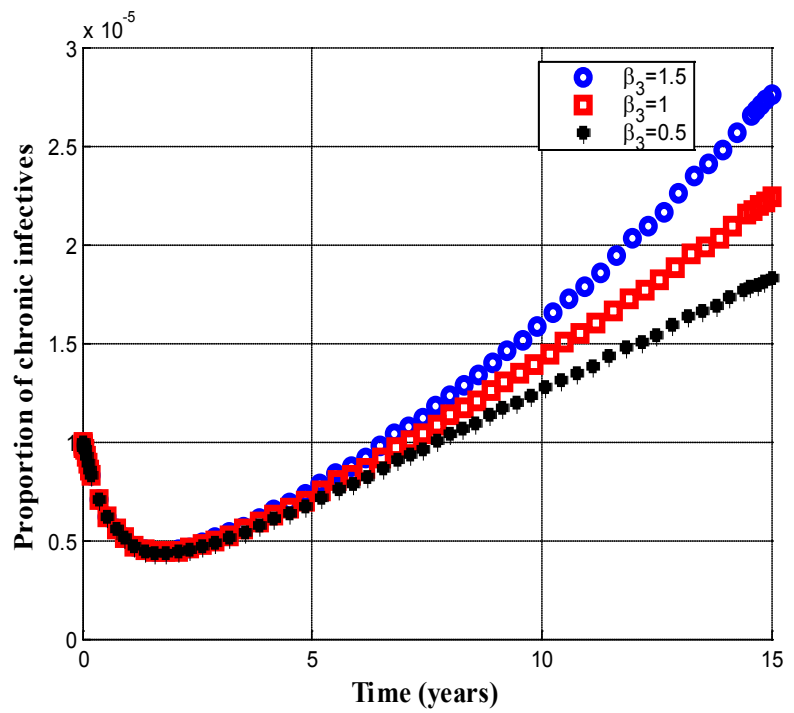

Figure 4.18. Variation of proportion of chronic infected population for different values of $\beta_{i}(i=1,2,3)$.

It is observed from figures. 4.17 and 4.18 , that when the effective contact rate of individuals still undergoing treatment $\beta_{3}$ become very small, the susceptible population increase and therefore the number of exposed individuals decrease, leading to the decrease of the acute and chronic infected individuals. As the rate of progression to acute infected class from exposed class $\varepsilon$ decrease, the exposed individuals who shifted to acute infected class decreases resulting to the decrease of the number of acute and chronic infective individuals in the community.

Figures 4.19 and 4.20, show the variation of proportion of chronic infectives and treated population for different recovery rates.

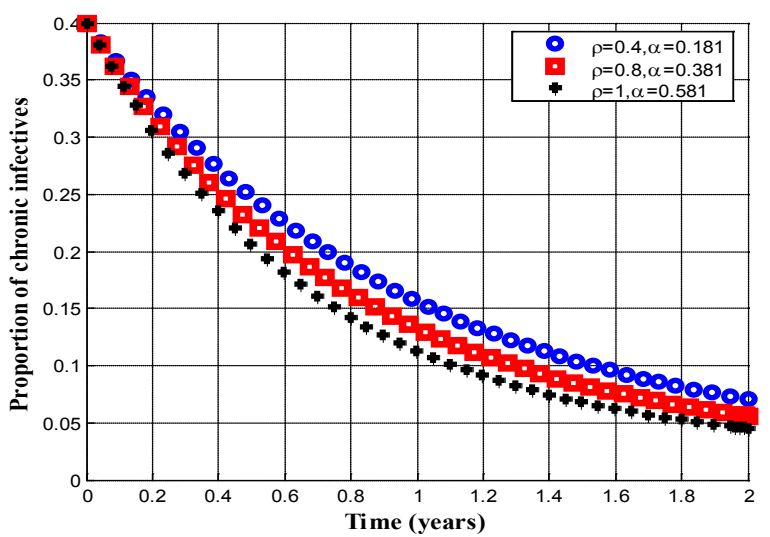

Figure 4.19. Variation of proportion of chronic infective population for different values of $\rho$ and $\alpha$.

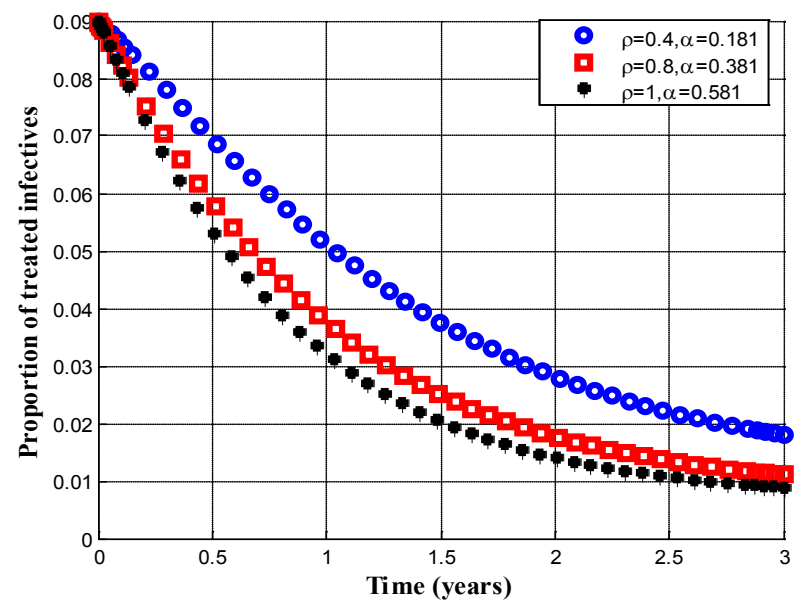

Figure 4.20. Variation of proportion of treated infective population for different values of $\rho$ and $\alpha$.

It is observed that as the recovery rates $\rho, \alpha$, increase, the chronic infectives decrease with time as seen in figure 4.19 leading to the decrease of treated group as seen in figure 4.20. This is an indication that, as the recovery rate increases, the rate of progression to treatment from acute infected and chronic infected groups $\pi_{1}$ and $\pi_{2}$ respectively decrease, resulting to the decrease of infected individuals (acute, chronic and treated ) in the community.

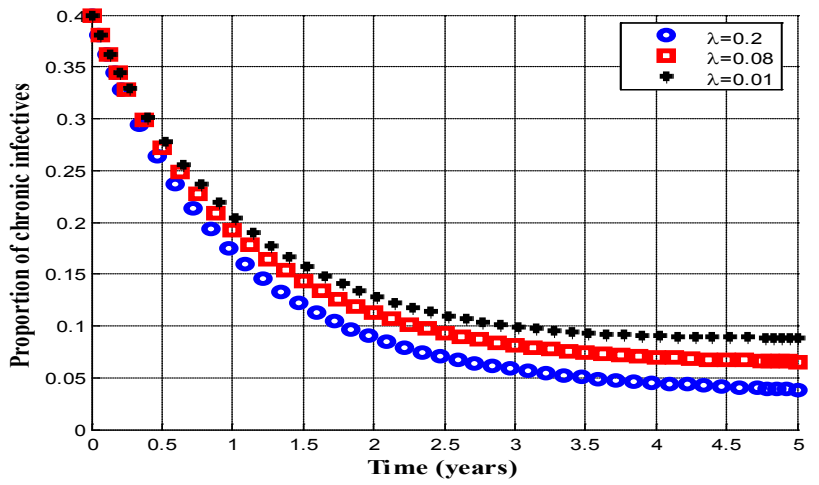

Figure 4.21. Variation of proportion of chronic infective population for different values of $\lambda$.

Figure 4.21 shows the variation of chronic infectives for different values of recruitment rate $(\lambda)$ of the population. It 
is clear from the figure that as the recruitment rate increases, the chronic infected population decrease. This might be due to the effect of treatment in the community which leads to the decline of infected individuals (acute, chronic and treated infectives) in the community.

\section{Discussions and Conclusions}

In this paper, a mathematical model of HCV showing the effects of treatment and infected immigrants on the spread of HCV disease with acute and chronic stages has been established. Both qualitative and numerical analysis of the model was done. The model incorporates the assumption that infected immigrants enter the homogenous population. It was shown that there exists a feasible region where the model is well posed in which a unique disease free equilibrium point exists.

The disease free and endemic equilibrium points were obtained and their stabilities investigated. A numerical study of the model has been conducted to see the effect of certain key parameters on the spread of the disease. It was observed that the disease become more endemic due to the presence of infected immigrants in the community. As the infected immigrants increase, the acute and chronic infective individuals also increase in the population. It was also shown that in the presence of treatment, the rate of infected individuals (acute and chronic) decrease and consequently the treated infected individuals decrease continuously. It was therefore shown that the disease can be eliminated at some point due to treatment and natural immunity of chronic infected individuals in the community.

Finally from the analysis, it may be hypothesized that preventive measures, through reducing rates of transmission of $\mathrm{HCV}$ are therefore necessary to the community. Reduced transmission leads to lower prevalence of the disease in the long-term. The national health cares to HCV should therefore seek to ensure that all people at risk or that have been at risk in the past, have access to and are supported in the use of $\mathrm{HCV}$ education and prevention services, regardless of their social and economic status.

\section{REFERENCES}

[1] J. Wales, L. Sanger, 2001. Acute hep C virus infection: Transmission, Diagnosis, Prevention and Treatment. Wikimedia Foundation, Inc
[2] L. A. Moyer, E. E. Mast, M. J. Alter, 1999, Hepatitis C: Prevention counseling and medical evaluation. American Academy of Family Physicians

[3] J. Wong, 2000,. "Silent killer". American Journal of Public Health", Vol 90

[4] C. Giordano, E. F. Drugts, G. Garber, C. Cooper, Evaluation of immigration status, race and language barriers on chronic hepatitis $\mathrm{C}$ virus infection management and treatment outcomes, Wolters Kluwer Health: Lippincott Williams and Wilkins, 2008

[5] A. Mehta, R. Pawson, S. Rajan, G. Hazlehurst, G. Dusheiko, R. Miller, P. Hewitt (1999) "Hepatitis C lookback programme: a single hospital experience", Transfusion Medicine , 9 (3) $189-193$

[6] G. L. Davis, K. Yalamanchili, S. Saadeh, R. Lepe, 2009, The prevalence of hepatitis $\mathrm{C}$ virus infection in Taxes: Implications for future health care. Baylcr University Medical centre

[7] L. Highleyman, 2010, "Chronic Hepatitis C Complications are Increasing, Especially among People Older than 60 Years". Gastroenterology

[8] C. D. Mazoff (2008), Disease Progression: Acute Hepatitis C. Alan Franciscus

[9] L. R. Fischer, D. H. Tope, S. Kathleen, R. N. Conboy, B. D. Hedblom, E. Ronberg, D. K. Shewmake, J. C. Butter, 2000. Screening for hepatitis $\mathrm{C}$ virus in a health maintenance organisatio, American Medical Association

[10] W. A. Zule, E. C. Costenbader, C. M. Coomes, W. M. Wechsberg, 2009. Effects of a hepatitis C virus educational intervention or a motivational intervention on alcohol use, injection drug use, and sexual risk behaviours among injection drug users. National centre for Biotechnology Information, U. S. National Library of Medicine, Bethesda MD, USA

[11] L. Cai, X. Li, A note on global stability of an SEI epidemic model with acute and chronic stages. Beijing Institute of Information Control, PR China, 2007

[12] G. M. Gomes, L. J. White, G. F. Medley, 2004, Infection, reinfection, and vaccination under suboptimal immune protection: epidemiological perspectives, J. Theor. Biol. 228, p. 539-549

[13] O. Diekmann, J. A. P. Heesterbeek and J. A. P. Metz, 1990, On the definition and computation of the basic reproduction ratio $R_{0}$ in the model of infectious disease in heterogeneous populations. Journal. Math. Biol. 2(1), 265-382

[14] A. Gumel, C. Castillo-Chavez, R. E. Mickens and D. P. Clemence, 2009, Simulation of the pertusis epidemiology in the united states: Effects of Adult booster doses, maths equations and application of Biology and industry, www.publc.asu.edu/.../pubs.htm retrieved on 9 April, 2011 